\title{
Burkitt Lymphoma Initially Presenting as Acute Pancreatitis in an Adolescent Boy
}

\author{
Hyemin Kim, Yiyoung Kwon, Eun Sil Kim, Hee Young Ju, Hong Hoe Koo, Yon Ho Choe and Mi Jin Kim \\ Department of Pediatrics, Samsung Medical Center, Sungkyunkwan University School of Medicine, Seoul, Korea
}

\begin{abstract}
In children and adolescents, acute pancreatitis is a rare cause of abdominal pain. The causes of pancreatitis in children are various including infection and drugs, but the overall cause of this condition in a pediatric patient is sometimes unknown. We describe a case of Burkitt lymphoma which showed acute pancreatitis findings as an initial presentation. In this case, a 16-year-old boy presented with abdominal pain in the left upper quadrant that had been present for one month. Pancreatitis was suspected due to high amylase and lipase and the computed tomography findings in the patient, which showed swelling and adjacent infiltration of the pancreas. However, initial treatments did not improve the patient's symptoms. The following imaging studies showed mass-like lesions involving the pancreas, distal duodenum and jejunum associated with mesenteric lymphadenopathy that suggested a lymphoma in this case. In the final analysis, the patient was diagnosed with Burkitt lymphoma which was seen on bone marrow biopsies and also found on the small bowel tissue biopsies.
\end{abstract}

Key Words: Pancreatitis, Burkitt lymphoma, Adolescents
pISSN 2233-5250 / eISSN 2233-4580 https://doi.org/10.15264/cpho.2020.27.2.124 Clin Pediatr Hematol Oncol 2020;27:124 128

Received on May 8, 2020 Revised on July 22, 2020 Accepted on August 30, 2020

Corresponding Author: Mi Jin Kim Department of Pediatrics, Samsung Medical Center, Sungkyunkwan University School of Medicine, 81 Irwon-ro, Gangnam-gu, Seoul 06351, Korea

Tel: +82-2-3410-0951

Fax: +82-2-3410-0043

E-mail: mijin1217.kim@samsung.com ORCID ID: orcid.org/0000-0002-4505-4083

\section{Introduction}

The experience of abdominal pain is one of common complaints of pediatric patients who go to an emergency department for treatment of this symptom. In children and adolescents, acute pancreatitis is one cause of abdominal pain. The most common causes of acute pancreatitis in children are idiopathic etiology, trauma, structural anomalies, multisystem disease, drugs, toxins, and viral infections [1,2]. However, lymphoma is noted as a rare cause of acute pancreatitis. In this study, we described a patient case of Burkitt lymphoma (BL), which showed acute pancreatitis findings as an initial presentation.

\section{Case Report}

A 16-year-old boy presented to our emergency room (ER) with a one-month history of abdominal pain in the left upper quadrant (LUQ). He described the pain of the first month as persistent dull ache and ranging in intensity from 3 to 4 on a visual analog scale (VAS). The pain was noted to have increased significantly for recent 3 days prior to the visit to the ER. He described this recent abdominal pain as a continuous squeezing pain ranging in intensity from 5 to 8 on VAS. He had aphthous stomatitis which occurred when he was tired, and had knee pain which was caused by exercise and was relieved by rest. He had no significant medical history including trauma, surgeries, and recent infections, and no drugs to take. He denied nausea, vomiting, diarrhea, weight loss, 
night sweat and fever. At the day before he came to our ER, esophagogastroduodenoscopy (EGD) and abdominal ultrasound were performed at another hospital, and the results were found to be within normal limits.

In our ER, his vital signs were blood pressure 145/73 $\mathrm{mmHg}$, heart rate $90 /$ minute, respiratory rate 20 /minute, and temperature $37.2^{\circ} \mathrm{C}$. His weight was $100 \mathrm{~kg}$, and height was $176.3 \mathrm{~cm}$. He experienced tenderness in his LUQ without rebound tenderness. A complete blood count showed white blood cells $8,480 / \mu \mathrm{L}$ (segmented neutrophils $58.4 \%$, lymphocytes $30.1 \%$, and monocytes 8.4\%), hemoglobin $8.3 \mathrm{~g} / \mathrm{dL}$ (MCV $83.4 \mathrm{fL}, \mathrm{MCH} 24.1 \mathrm{pg}$ ) hematocrit $28.7 \%$, and platelets $616,000 / \mu \mathrm{L}$. He had hypoalbuminemia and a reduced level of serum protein (albumin $3.1 \mathrm{~g} / \mathrm{dL}$, total protein $5.3 \mathrm{~g} / \mathrm{dL}$ ). The C-reactive protein was elevated at $5.08 \mathrm{mg} / \mathrm{dL}$ (normal, $<0.5 \mathrm{mg} / \mathrm{dL}$ ), and ferritin was $9.0 \mathrm{ng} / \mathrm{mL}$. The amylase and lipase were $161 \mathrm{U} / \mathrm{L}$ and $411.7 \mathrm{U} / \mathrm{L}$, respectively. His electrolyte profile, alkaline phosphatase, aspartate aminotransferase, alanine aminotransferase, and bilirubin were noted as normal. An initial abdomen computed tomography (CT) scan showed swelling and adjacent infiltration of pancreas, minimal amount of ascites, and no other abnormalities were found in the small and large bowel (Fig. 1). Based on these results, he was admitted with impression of acute pancreatitis, and we started conservative treatment with intravenous hydration, analgesics, gabexate and given nothing by mouth.

After admission, since the symptoms were not relieved and the hemoglobin was decreased, EGD and colonoscopy were performed to evaluate gastrointestinal
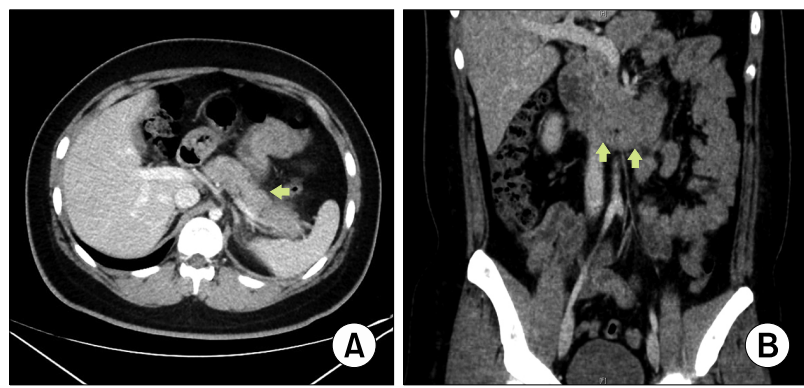

Fig. 1. (A, B) initial abdomen computed tomography (CT) scan showed swelling and adjacent infiltration of the pancreas. bleeding. A peripheral blood smear (PBS) examination was also performed. EGD revealed duodenitis and colonoscopy were shown to be within normal limits. Urease breath test was negative. The PBS examination was shown microcytic and hypochromic anemia. According to these results, he was considered to be more likely to have iron deficiency anemia and decided to take iron supplements if oral administration was possible. Because amylase and lipase levels that had risen to $236.8 \mathrm{U} / \mathrm{L}$ and $748.5 \mathrm{U} / \mathrm{L}$ on the 2nd day of hospitalization fell to $110.8 \mathrm{U} / \mathrm{L}$ and $360.8 \mathrm{U} / \mathrm{L}$ on the 12th day, and the symptoms improved, he began to soft bland diet (SBD). However, then his abdominal pain worsened. He started fasting again on the 15th day. Next, a fecal calprotectin test was performed for the point of differentiating inflammatory bowel disease (IBD), which could induce pancreatitis. His calprotectin was shown to have been elevated to more than $1,000 \mu \mathrm{g} / \mathrm{g}$.

On the 17th day of hospitalization, because of persistent abdominal pain, elevation of amylase (177.7 U/L) and lipase (611.7 U/L) levels, and low serum albumin (albumin $3.4 \mathrm{~g} / \mathrm{dL}$, total calcium $9.0 \mathrm{mg} / \mathrm{dL}$ ), we checked a follow-up CT scan. The CT scan showed acute pancreatitis, with mass-forming bowel wall thickening in the distal duodenum with mesenteric fat infiltration and large enhancing lymph node (LN) nearby unusual large enhancing LN (Fig. 2). For further evaluation of the LN enlargement, a magnetic resonance imaging (MRI) was performed, and it showed multiple T2 isosignal mass-like lesions (showing diffusion restriction) involving the pancreas, distal duodenum and jejunum associated with a mesenteric lymphadenopathy that suggested a lymphoma (Fig. 3). A fluorodeoxyglucose (FDG)-positron emission tomography (PET) was performed for the next evaluation. It was shown that multiple hypermetabolic malignant lesions in the small bowel, mesentery LNs, pancreas and right iliac bone were noted (Fig. 4). On the 27th day, for confirmatory diagnosis, we performed bone marrow (BM) biopsies and tissue biopsies by the small bowel resection. Pathologic diagnosis of BM and small bowel were BL. There were two fungating masses measuring $2.6 \times 2 \mathrm{~cm}$ and $6 \times 5.6 \mathrm{~cm}$ in the small bowel specimen which invade 

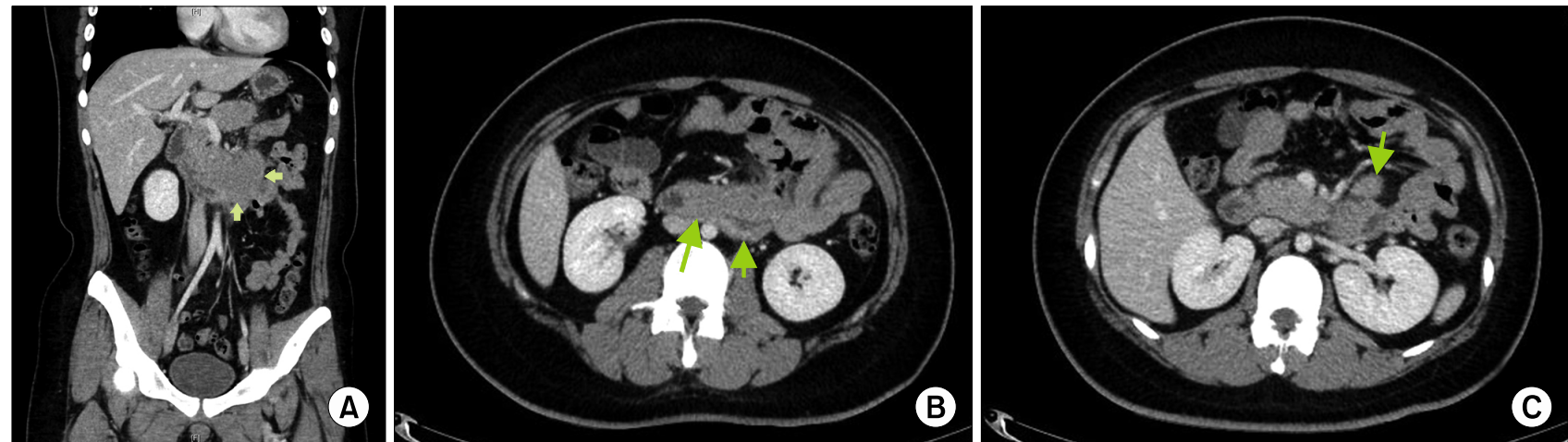

Fig. 2. Follow-up CT scan on the 17th day of the hospitalization; (A) CT scan showed a more swollen pancreas than the initial CT scan. It is considered as a persistent acute pancreatitis. Also, this CT scan showed a mass-forming bowel wall thickening at the duodenum 3rd and 4th portions and duodenojejunal junction. (B) Bowel wall thickening was suspected in the jejunum. (C) Unusual large enhancing lymph node was seen around the area of the thickened bowel wall.
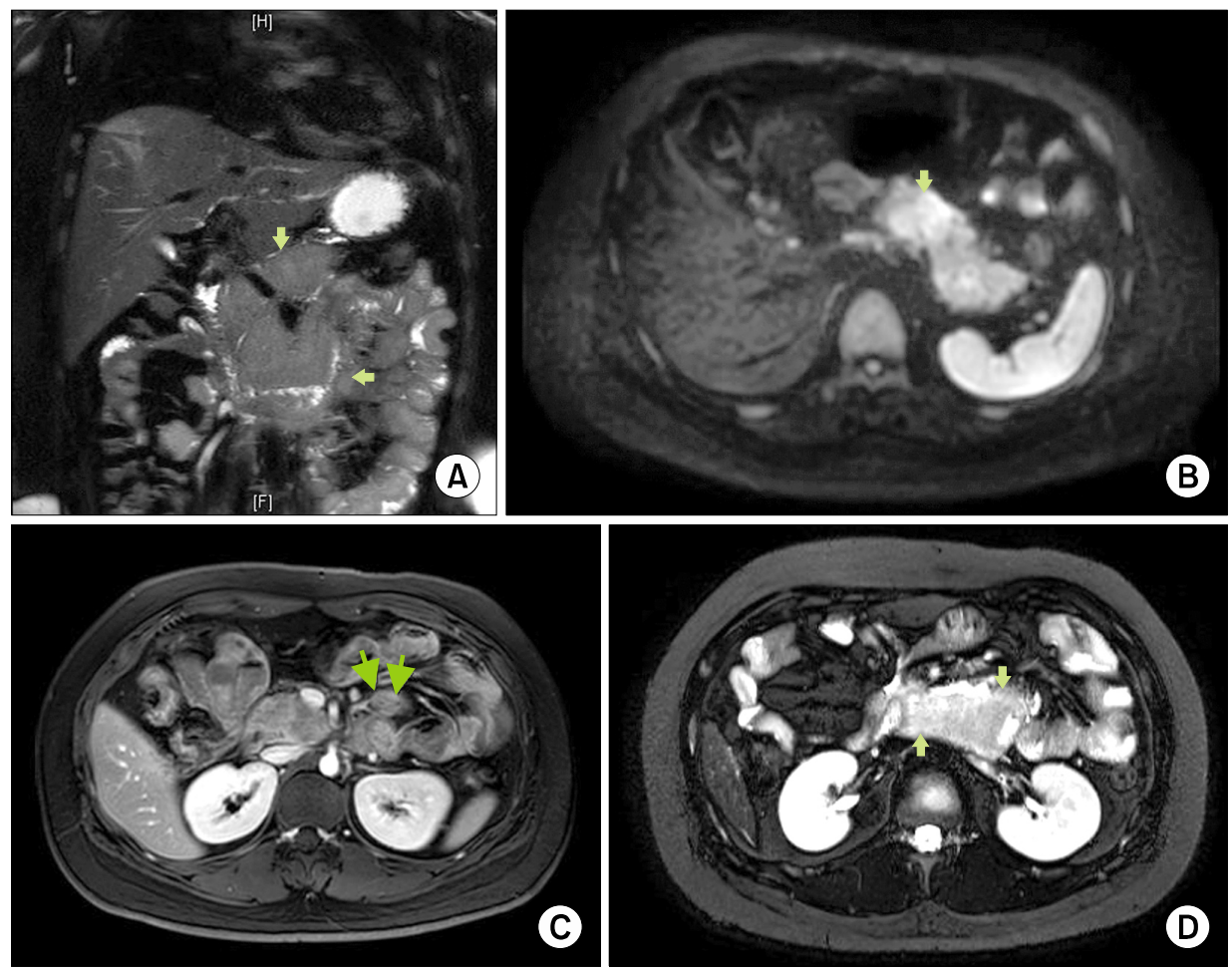

Fig. 3. MRI on the 24th day of the hospitalization; (A) Multple T2 isosignal mass-like lesions (showing diffusion restriction) involving the pancreas, distal duodenum and jejunum associated with mesenteric lymphadenopathy. (B) In the diffusion weighted image, there are mass-like lesions of variable size across the pancreas head, uncinate process, body, and tail, with diffusion restriction. (C, D) Multifocal and homogeneous $\mathrm{T} 2$ isosignal masslike lesions in the small bowel with mesenteric lymphadenopathy. muscularis propria. In the BM biopsies, the involvement of $\mathrm{BL}$ was shown at the right side, but not the left side. Immunohistochemical testing was positive for $\mathrm{CD} 3$, CD10, CD20, Ki-67, and BCL-6, and focal weak positive for BCL-2. The test was negative for TdT. The results of the in-situ hybridization test of Epstein-Barr virus were negative. Fluorescent In-Situ Hybridization revealed 14.0\% of cells with IGH/MYC rearrangement and $11.5 \%$ of cells with trisomy 1q. Thereafter, it was confirmed that the fi- nal diagnosis of this patient was BL, in stage IV.

After being diagnosed with BL, on the 35 th day of hospitalization, he was given chemotherapy. He started SBD on the first day of the chemotherapy, and regular diet on the 7 th day of the chemotherapy. The follow-up MRI after the first chemotherapy showed a markedly improved state of lymphoma involving the pancreas, distal duodenum and jejunum which was associated with a mesenteric lymphadenopathy. He received eight cycles 


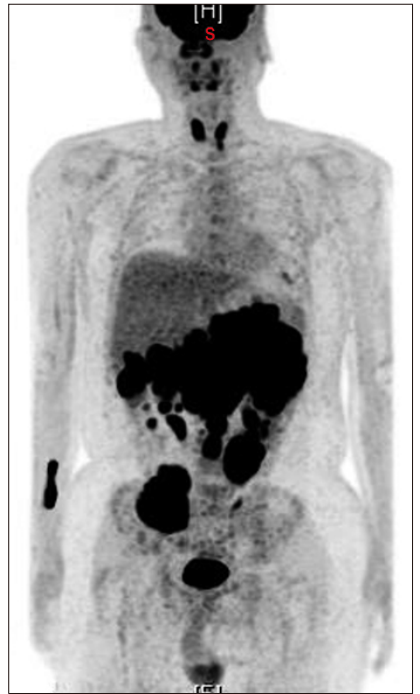

Fig. 4. FDG-PET; multiple hypermetabolic malignant lesions in the small bowel, mesentery lymph nodes, pancreas, Rt. iliac bone, and probably both thyroid glands.

of chemotherapy, rituximab and LMB protocol, consisting of COP (vincristine, cyclophosphamide, prednisolone, and intrathecal methotrexate, and cytosine arabinoside), R-COPADM (rituximab, methotrexate, doxorubicine, and COP), and R-CYVE (rituximab, cytosine arabinoside, and etoposide) for 6 months, and no radiotherapy and hematopoietic stem cell transplantation There were no significant complications during chemotherapy. Up to now, 10 months after treatment, he is receiving monthly outpatient care without sign of cancer recurrence and metastasis.

\section{Discussion}

The case of pancreatitis is not a common cause of abdominal pain whereas it is associated with significant morbidity and mortality. There are numerous conditions that can lead to pancreatitis. However, the case of lymphoma as a cause of pancreatitis is considered to be a rare occurrence in pediatric patients [3].

Non-Hodgkin lymphoma (NHL) often involves many organs, and clinical presentation varies according to involved sites. It is noted in several studies that the pancreas, however, is an uncommon site [4-6]. In these studies, the imaging findings include diffuse enlargement of the pancreas, focal homogeneous masses, or the presence of both conditions.

The clinical presentation and outcome of the pediatric patients who have NHL involving the pancreas represent the conditions of abdominal pain, jaundice, elevated pancreatic enzyme, or gastrointestinal symptoms which is similar to the case of pancreatitis [7-9]. Our patient did not show jaundice although there was a showing of abdominal pain, elevated pancreatic enzyme and gastrointestinal symptoms with CT scan evidence of swelling and adjacent infiltration of pancreas. His history of aphtous stomatitis and knee pain and his elevated fecal calprotectin level made us consider IBD as his disease. Therefore, we performed follow-up CT scan after IBD work up. In this sense, it should be noted that the mass may not be visualized radiologically at initial presentation, but rather will present with acute pancreatitis dominated by significant inflammatory changes [3]. Based on the clinical findings from our case, we suggest that follow-up cross-sectional imaging studies be performed for a more accurate clinical diagnosis.

The cross-sectional imaging studies have been used to help diagnose and evaluate disease severity. In some cases, a differentiation between pancreatitis and tumor involvement of pancreas may be difficult to distinguish [10], because the inflammatory changes caused by acute pancreatitis can mask the underlying tumor [3]. The lesion may be detected only on follow-up scans after the inflammation has subsided.

Still, it is noted that BL can be treated with chemotherapy. In children, the prognosis is good with survival rates $>90 \%$ [11]. As BL has doubling time of about 24 hours, which is the shortest among human neoplasms $[12,13]$, it requires a prompt diagnosis for initiation of the treatment for the best patient outcomes.

Our patient was obese at the time of admission. Several studies have found the association between obesity in childhood and cancer risk [14,15]. These studies showed that being obese increased the risk of cancer and that the more severe the obesity, the more likely it tends to develop cancer early. However, in these studies, the average age at diagnosis was about 40 which was differ- 
ent from our patient. Therefore, further research on the relationship between malignancies diagnosed with adolescence and obesity is needed.

In conclusion, although uncommon, if the patient had persistent symptoms after the initial treatment, physicians should consider the differential diagnosis of tumorous condition even in pediatric patients.

\section{Conflict of Interest Statement}

The authors have no conflict of interest to declare.

\section{References}

1. Benifla M, Weizman Z. Acute pancreatitis in childhood: analysis of literature data. J Clin Gastroenterol 2003;37:169-72.

2. Suzuki M, Sai JK, Shimizu T. Acute pancreatitis in children and adolescents. World J Gastrointest Pathophysiol 2014;5: 416-26.

3. Shanbhogue AK, Fasih N, Surabhi VR, Doherty GP, Shanbhogue DK, Sethi SK. A clinical and radiologic review of uncommon types and causes of pancreatitis. Radiographics 2009;29:100326.

4. Ng YY, Healy JC, Vincent JM, Kingston JE, Armstrong P, Reznek RH. The radiology of non-Hodgkin's lymphoma in childhood: a review of 80 cases. Clin Radiol 1994;49:594-600.

5. Vade A, Blane CE. Imaging of Burkitt lymphoma in pediatric patients. Pediatr Radiol 1985;15:123-6.
6. Carbonetti F, Iannicelli E, Federici M, et al. Primary pancreatic Burkitt lymphoma presenting as acute pancreatitis. J Gastrointest Cancer 2014;45 Suppl 1:265-9.

7. Bacchi LM, Ucella I, Amancio TT, Gonçalves MC, Palhares RB, Siqueira SAC. Burkitt lymphoma mimicking acute pancreatitis. Autops Case Rep 2012;2:5-11.

8. Biko DM, Anupindi SA, Hernandez A, Kersun L, Bellah R. Childhood Burkitt lymphoma: abdominal and pelvic imaging findings. AJR Am J Roentgenol 2009;192:1304-15.

9. Raj M, Ghoshal UC, Choudhuri G, Mohindra S. Primary gastric lymphoma presenting as acute pancreatitis: a case report. JOP 2013:14:463-5.

10. Amodio J, Brodsky JE. Pediatric Burkitt lymphoma presenting as acute pancreatitis: MRI characteristics. Pediatr Radiol 2010; 40:770-2.

11. Mukhtar F, Boffetta P, Risch HA, et al. Survival predictors of Burkitt's lymphoma in children, adults and elderly in the United States during 2000-2013. Int J Cancer 2017;140:1494502.

12. Richman SD, Appelbaum F, Levenson SM, Johnston GS, Ziegler JL. 67Ga radionuclide imaging in Burkitt's lymphoma. Radiology 1975;117:639-45.

13. Ferry JA. Burkitt's lymphoma: clinicopathologic features and differential diagnosis. Oncologist 2006;11:375-83.

14. Leiba M, Leiba A, Keinan-Boker L, et al. Adolescent weight and height are predictors of specific non-Hodgkin lymphoma subtypes among a cohort of 2,352,988 individuals aged 16 to 19 years. Cancer 2016;122:1068-77.

15. Furer A, Afek A, Sommer A, et al. Adolescent obesity and midlife cancer risk: a population-based cohort study of $2 \cdot 3$ million adolescents in Israel. Lancet Diabetes Endocrinol 2020;8:216-25. 